\title{
EFFECT OF SOME PLANT EXTRACTS AND OILS ON GRAPE DOWNY MILDEW DISEASE
}

\author{
M. M. Ammar, G. A. Amer, A. M. Abd El-All and A. E. Badawy \\ Agriculture Botany Department, Faculty of Agriculture, Menoufia University, Egypt.
}

Received: Oct. 8, 2017

Accepted: Nov. 26, 2017

\begin{abstract}
The present study was conducted in 2015 and 2016 to find out the best ecofriendly method(s) to control downy mildew disease of grapes. Water extracts of castor, oleander and mint significantly reduced the disease incidence, increased chlorophyll and carotenoids content, improved plant water relations and increased yield production. The same observations were obtained using emulsion oils of castor, thyme and mint. Castor water extract and emulsion oils showed the best results, all treatment of plant oils and plant extracts caused a significant remarkable increase in no. of clusters, cluster length and yield per plant.
\end{abstract}

Key words: Downy mildew, Plasmopara viticola ,plant oils , plant extracts, photosynthetic pigments, water relations and yield production.

\section{INTRODUCTION}

Table grapes are one of the most widelygrown fruit crops in Egypt. It is considered to be the second most important fruit crop after citrus. Egyptian geographical spread of production enables fresh sweet grapes to be available from May to July. Table grapes are grown from Alexandria in the north of Egypt to Aswan in the south. There are many varieties of table grapes produced in Egypt, like Early sweet, Superior, Thomoson, Flame Seedless, Crimson, and Red globe. There are clear competitions among the Egyptian growers. every year because of the new grape cultivars production. So the only thing that keeps one ahead of others in the market is the ability of producing high quality grapes due to following Good Agriculture Practices The cultivated total area with grape during 2016 season in Egypt was approximately 167,048 feddans produced about $1,200,000$ tons of grape with average 9 tons / feddan (according to Economic Affairs sector - Ministry of Agriculture and Land Reclamation, Egypt, 2016).

Some diseases attack grape plants during the different growth stages causing great yield loses. Out of them, Grey mold caused by Botrytis cinerea, Powdery mildew caused by Uncinula necator, Black rot caused by Guigardia bidwelli and Downey mildew caused by Plasmopara viticola (Berk $\&$ Curt.). Downy mildew is considered very important disease, where, it causes big problem to grapes. This study aimed to find out environmental friend control methods for such disease and have positive physiological and productive effects. In this work, some plant water extracts. plant oils, biocontrol agents and some recommended fungicides were tested to achieve the best way for controlling grape downy mildew disease. Out of them, downy mildew (ODM) caused by plasmopara viticola (Berk.) Achimu and Schloseer (1992) found that spraying fine mist of $A$. indica seed extracts or 1 of 3 commercial neem products $2 \mathrm{~d}$ before inoculation with plasmopara viticola gave significant reduction in disease severity with an efficacy of $>90 \%$. They attributed the antifungal properties of neem products to the inhibition of the indirect germination of sporangia. Dai et al., (1994) mentioned that crude foliar extracts of grape resistant cultivar had a stronger antifungal activity than those of the intermediate and susceptible genotypes. In response to infection by $P$. viticola, flavonoids appeared at an early stage in the resistant cultivar, which may be play an important role in its resistance. Klinkenberg et al., (1998) 
indicated that induced resistance can be an additional tool in the integrated control of diseases caused by Oomycetes. Robtic et al., (2000) used mixture of essential and aromatic oil extracts (Urticum) to control downy mildew of grapes which provided better control to the disease (90.8 to $97.1 \%$ ). Berkelman_ Lohnertz (2002) conducted laboratory and field trials to evaluate the efficacy of plant extracts and inorganic, naturally occurring compounds to control downy mildew (P.viticola). Chen Jiao et al., (2002) determined the inhibitory effect of 58 plant extracts against sporangial germination and their effective control of grape downy mildew (P.viticola) which some of them inhibited the pathogen with high inhibition rates. They also mentioned that the effects of 5 plant extracts were the same or better than that of traditional fungicide liquid Bordeaux. Cohen et al., (2002) indicated that extracts of the shoots of Inula viscosa (Basin) exhibited a strong fungicidal activity against $P$. viticola and other pathogens. They also mentioned that thin layer chromatography analyses revealed at least 7 fungicidal compounds.

Schmitt et al., (2002) reported that single or combined application of $R$. sacalinensis and B.brevis plant extracts resulted in lower disease incidence $(P$. viticola), under field conditions .Schilder et al.,(2002) evaluated various environmental friendly products in vineyards in Michigan USA for their effecacy in controlling downy mildew (P.viticola). They mentioned that JMS stylet oil , Armicarb , Serenade, AQ10, Elexa and Milsana provided moderate control of downy and powdery mildew .Scannavini et al., (2004) in their field trials in Italy provide that plant extracts and the growth regulator (Kendal) stimulate grape plant development and natural disease resistance with cooper and dithiocarbamate fungicides at reduced concentrations and confirmed the results of downy mildew control. Cohen et al., (2006) reported that paste extracts of Inula Viscosa leaves and it's emulsified oil provided excellent activity against grape downy mildew in the field. The disease in treated shoots was below $0.125 \%$ (paste in water). Kast and Bleyer (2006) examined the efficacy of 23 plant extracts against grape downy mildew (P.viticola). They mentioned that the greatest inhibition of fungal growth obtained with extracts from Hedra helix (leaves in water). They also found that the antifungal effect increased as the extract concentration increased and the reduction in disease severity was $70-90 \%$. Chovelon (2006) used plant extract (My cosine) against downy mildew of grapes in Italy. He mentioned that Salix extract was a promising alternative to copper. Rajeswari et al., (2008) revealed that the neem seed kernel extract at $5 \%$ significantly inhibited the sporangial germination $(75.36 \%)$ of $P$.viticola and effectively inhibited the disease development. Dagostin et al., (2009) applied sage (Salvia officinales) extract to artificially inoculated potted grapevine plants under greenhouse conditions which provided high control of the disease. Gindro and Godard (2009) evaluated the efficacy and mode of action of various elicitors (i.e. activators of defence mechanisms) on detached leaves, as on whole plants of Vitis vnifera. The results showed that root extract of rhubarb (Rheum palmatum), bark extract of glossy bukthorn (Frangula alnus) and leaf extract of Aloe (Aloe vera) stimulated the synthesis of aviniferin (the most toxic stilben for P.viticola) and inhibited the development of the pathogen at a significant degree Dogastin et al.,(2010) observed that sage extract provided a high level of sustained downy mildew (P.viticola) disease control, under green house and field conditions .

Pereira et al., (2010) used plant extracts beside traditional fungicides for the protection of vine (Vitis vinifera) against downy mildew (P.viticola) .Molitor et al. , (2010) indicated that water- extract of the chinese Soapberry (Sapindus mukorossi) gave an excellent efficacy against $P$.viticola . Harm et al. (2011) mentioned that chemical and natural resistance - inducing substanses may be used to decrease the 
susceptibility of plants to various pathogens. They also reported that the invoked mode of induced resistance was verified to control downy mildew on grape vine plants, which was determined by the increase of pathogenesis - related proteins. Of the candidate resistance inducers Solidago canadensis (Can G) extract which peovided a protection more than $80 \%$. leite et al., (2011) indicated that garlic extract and vegetable oils were effective in controlling DM disease of grape in Brazil. Rawal (2013) used plant extracts to control DM disease of grapes in India. Torre et al., (2014) revealed that essential oils, BM-608 and Sporatec were able to control the development of grapes downy mildew. Anelkovic et al., (2015) determined the phenolic composition of the grapevine leaf extract and their antimicrobial activity. They noticed that total phonlic content was higher in healthy grapevine leaf extracts than infected grapevine leaf extracts. The first was richer in flavonls, phenolic acid and flavan -3-ols. Naik et al., (2015) conducted field experiments to assess effect of aqueous leaf extract of Agave cantala on oxidative mechanism in the leaves of Vitis vnifera infected with downy mildew. Enhasments of chlorophylls, carotenoids with total polyphenols were noticed in grape leaves infected downy mildew after application of A.cantala leaf extract. Further, it was also noticed that activities of enzymes peroxidase and polyphenol oxidase were increased and activity of enzyme catalase was decreased in sprayed grape leaves. They conc luded; from these results; that Agava cantala leaf extract may be useful for controlling downy mildew of grape.

\section{MATERIALS AND METHODS}

These experiments were conducted in a private farm at Tanbol, Sadat city, Menoufia governorate, Egypt, at 2015 and 2016 growing seasons. Both Crimson cv (5 years old) and Flame cv. (6 years old) grape plants were subjected to different individual treatments in order to control grape downy mildew. A complete randomized block design system with three replicates per each treatment was followed. Each replicate had 25 grape well grown plants $(2 \times 3$ meters space). Drop irrigation system and the recommended fertilization were applied. The plants were left to the natural inoculation conditions; the treatments were applied six times, the first treatments was applied at the frist of March and repeated each 15 days up to the middle of May. Each application required 20 liter (each litre contents of 2,4 and $6 \mathrm{ml} / \mathrm{l}$ of plant extracts and 2, 3.5, and $4.5 \mathrm{ml} / \mathrm{l}$ of plant oils)of the compound per replicate (25 plants) and control treatments were sprayed with sterilized distilled water. A total of 54 treatments were carried out for each grape cultivar as following:

\section{1-Plant extracts:}

Hundred grams of castor, mint and/or nerium leaves were seperatly boiled in enough amount of distilled water for half an hour. The extracts were filtered through Watman No 1 filter papers and completed for one liter with sterilized distilled water (10\% conc.). The concentrations of 2,4 and $6 \mathrm{ml} / \mathrm{l}$ of each extract were adopted and sprayed ; individually on grape plants

\section{Plant oils:}

Oils of castor, mint and thyme were obtained from El-Gomhoria Company for Oils and Pharmaceutical Industries, Cairo, Egypt. The oils were emulsified with $3 \%$ Tween 80. Oil emulsion was sepcralty sprayed six times (every15days) at the concentrations of $2.0,3.5$ and $4.5 \mathrm{ml} / \mathrm{l}(20$ liters/ replicate).

Data recorded: The results of severity of infection, growth characteristics, photosynthetic pigments, permeability, transpiration rate, osmotic pressure, water relations and yield production as affected with the different treatments were estimated as following: 
1. Severity of infection: Severity of infection was determined four times using the scale of 0-9 according to the area of the leaves covered with the disease symptoms where: (O: healthy plants and 9: up to 100\%). The formulas of Soliman et al., (1988) were used for this estimation as following:

Severity of infection $=(a \times b) /(N \times K) \times 100$ Where:

$a=$ Number of the diseased plants

$\mathrm{b}=$ Infection rate (0-9)

$\mathrm{N}=$ Total number of the plants/plot

$\mathrm{K}=$ Total infection rates

\section{Physiological aspects:}

\subsection{Photosynthetic pigments:}

Chlorophyll a, b and carotenoids were determined from fresh leaves, using spectrophotometer method reported by Fadeel (1962) as follow: a known fresh weight of leaves $(0.5 \mathrm{~g})$ was homogenized in $15 \mathrm{ml}$ of $85 \%$ aqueous acetone for 10 minutes. The homogenate material was centrifuged and the supernatant was made up to the volume of $5 \mathrm{ml}$ with $85 \%$ acetone then measured at three wave lengths of 662 , 644and $440 \mathrm{~nm}$ using SPEKOL spectrophotometer (Model: SP/02 DP). Pigment concentrations were calculated according to the following equations:

Chl, $\mathrm{a}=9.784 \times \mathrm{E} 662-0.99 \times \mathrm{E} 644(\mathrm{mg} / \mathrm{l})$ Chl, $b=21.426 \times E 644-4.63 \times E$ 662(mg/l) Carotenoids $=4.695 \times \mathrm{E} 440-0.268(\mathrm{a}+\mathrm{b})$ $(\mathrm{mg} / \mathrm{l})$. The concentration of pigments was then expressed in $\mathrm{mg} / \mathrm{g}$ D. W.t.

\subsection{Water Relations:}

The total water content in leaves were determined using the methods described by Gosev (1960). Relative water content (RWC) and leaf water deficit (LWD): Equal leaf discs $(1 \mathrm{~cm})$ were cut from 90 days old plants, weighed to give the fresh weight (FW) floated on water for 6 hours until they reweighed (turgidity weight) and final oven dried at $70{ }^{\circ} \mathrm{C}$ for 72 hours to reach a constant weight. Relative water content and leaf water deficit (LWD) were calculated using the following formula according to Kalapos (1994):

RWC $\%=[($ Turgid weight - Fresh weight $) /$ (Turgid weight - Dry weight)] $\times 100$

LWD \% $=100-$ RWC

\section{Yield production:}

The effects of various treatments to control downy mildew disease of grapes on yield components were studied. Such studies included the average number of clusters per plant, average cluster length $(\mathrm{cm})$ and the average yield/plant $(\mathrm{kg})$.

\section{RESULTS}

Under field and natural inoculation conditions; both Crimson and Flame grape cultivars were subjected to some ecofriendly methods to control downy mildew disease. These methods included water plant extracts, plant oils and biocontrol agents, integration of plant extracts and biocontrol agents and specific fungicides in comparison. These trials were conducted both at 2015 and 2016 growing seasons.

\section{Effect of plant water extracts and plant oils:}

\subsection{A. (plant extracts):}

Severity of infection was also significantly affected by the application of plant water extracts (Table 1). Castor leaf extract was more efficient than those of oleander and mint ones. As example, severity of infection at June 2015 recorded 8.5, 5.2, 3.2 and $19.2 \%$ on Crimson cultivar when $2,4,6 \mathrm{ml} / \mathrm{l}$ of castor plant extracts were applied and the untreated control plants; respectively . In the same respect; severity of infection was 7.8, 5.5, 2.8 and $15.7 \%$ on Flame cultivar. At September 2015; Severity of infection recorded 18.8, 10.2, 7.2 and $40.8 \%$ for castor treatments and control plants; respectively. At the same month; these were $16.8,10.4,6.4$ and $37.8 \%$ respectively on Flame cultivar. Nearly similar results were obtained at the second year of investigation (2016). 


\begin{tabular}{|c|c|c|c|c|c|c|c|c|c|c|c|c|c|}
\hline \multirow{8}{*}{$\begin{array}{l}\text { 등 } \\
\stackrel{0}{E} \\
\text { 은 }\end{array}$} & \multirow{4}{*}{$\stackrel{0}{\stackrel{0}{ }}$} & 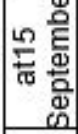 & $\hat{\stackrel{N}{ }}$ & $\stackrel{20}{\circ}$ & $\begin{array}{l}\infty \\
\emptyset\end{array}$ & $\stackrel{2}{\approx}$ & I & $\stackrel{m}{\circ}$ & $\stackrel{\overbrace{}}{\tilde{N}}$ & $\stackrel{\infty}{\sim}$ & $\stackrel{m}{\square}$ & m & N \\
\hline & & 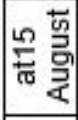 & $\stackrel{N}{\sim}$ & ণ் & $\bar{\sigma}$ & ¿े & $\stackrel{N}{m}$ & $\begin{array}{l}\infty \\
\sigma^{\circ}\end{array}$ & $\begin{array}{l}\infty \\
\stackrel{\sim}{N}\end{array}$ & $\stackrel{+}{\sigma}$ & $\stackrel{\infty}{\stackrel{\infty}{\sim}}$ & io & $\stackrel{L}{\sim}$ \\
\hline & & $\sum_{i}^{\frac{n}{\pi}} \frac{2}{3}$ & $\underset{\leftarrow}{ \pm}$ & $m$ & N & $\stackrel{\sim}{\oplus}$ & ㅁ. & ๙̃ & $\stackrel{\infty}{\infty}$ & $\stackrel{\varphi}{\varphi}$ & $\stackrel{\sim}{\sim}$ & 品 & $\stackrel{d}{\sim}$ \\
\hline & & 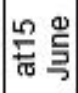 & $\stackrel{\sigma}{\pi}$ & $\stackrel{\sigma}{\forall}$ & गे & ஸे & Lึ & 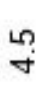 & $\stackrel{L}{\square}$ & $\stackrel{\circ}{\circ}$ & ᄂ? & ํ. & $\stackrel{\circ}{N}$ \\
\hline & \multirow{4}{*}{ 눙 } & 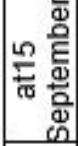 & $\begin{array}{l}\infty \\
\infty \\
-\end{array}$ & $\stackrel{\sim}{\circ}$ & $N$ & ํ. & $\stackrel{50}{=}$ & $\stackrel{\infty}{\infty}$ & $\frac{n}{\sim}$ & $\stackrel{\varphi}{\sigma}$ & $\begin{array}{l}\infty \\
\dot{J}\end{array}$ & $\begin{array}{l}\infty \\
\stackrel{\circ}{q}\end{array}$ & 오 \\
\hline & & 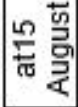 & $\stackrel{\sigma}{\sim}$ & $\stackrel{\sim}{\sigma}$ & $\overrightarrow{6}$ & $\stackrel{\infty}{\sim}$ & $\stackrel{\square}{\circ}$ & ஸे & ণ্ & $\stackrel{N}{\sim}$ & $\stackrel{N}{\sim}$ & $\stackrel{m}{\infty}$ & ָก \\
\hline & & $\sum_{\pi}^{\infty}$ & $\stackrel{\sim}{\sigma}$ & $\hat{\infty}^{\prime}$ & vं & $\stackrel{+}{=}$ & $\stackrel{N}{\sigma}$ & $\begin{array}{l}\infty \\
\infty\end{array}$ & $\stackrel{\sim}{\sigma}$ & $\stackrel{\nabla}{\bullet}$ & $\stackrel{n}{\leftarrow}$ & ผn & m \\
\hline & & 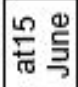 & $\omega^{\infty}$ & นู & $\underset{m}{N}$ & $\tilde{\sigma}$ & نे & $\stackrel{\infty}{+}$ & $\stackrel{\nabla}{\circ}$ & $\underset{N}{N}$ & $\underset{\infty}{\sim}$ & Nָ & $\stackrel{d}{N}$ \\
\hline \multirow{8}{*}{ 离 } & \multirow{4}{*}{$\stackrel{\oplus}{\stackrel{0}{N}}$} & 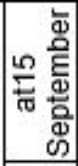 & $\stackrel{\infty}{\infty}$ & ํ. & $\underset{\omega}{N}$ & مै & $\stackrel{2}{=}$ & $\begin{array}{l}\varphi \\
\sigma^{\prime}\end{array}$ & 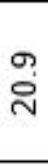 & 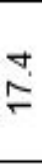 & $\stackrel{\sim}{\sim}$ & సָ & $\stackrel{\circ}{m}$ \\
\hline & & 边苛 & $\stackrel{N}{\circ}$ & $\underset{\infty}{\sim}$ & $\stackrel{N}{\omega_{0}^{\prime}}$ & $\begin{array}{l}\infty \\
\varphi \\
\omega\end{array}$ & $\stackrel{m}{\circ}$ & $\widehat{N}_{\infty}$ & $\begin{array}{l}\nabla \\
\sigma\end{array}$ & $\stackrel{N}{\Sigma}$ & $\hat{\circ}$ & $\stackrel{\nabla}{\mathrm{N}}$ & $\underset{m}{\forall}$ \\
\hline & & $\sum_{\pi}^{\infty} \frac{0}{\pi}$ & $\stackrel{\oplus}{\sim}$ & م & 5 & $\underset{⿱}{ \pm}$ & $\hat{\sigma}$ & $m$ & $\stackrel{\sim}{\sim}$ & $\stackrel{n}{\square}$ & ֶ. & กั & ñ \\
\hline & & 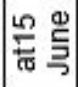 & m. & $\stackrel{4}{\forall}$ & m. & $\begin{array}{l}\stackrel{0}{0} \\
\infty\end{array}$ & $\underset{\text { L }}{N}$ & $\bar{\nabla}$ & $\stackrel{\sim}{\sim}$ & ஸे & $\begin{array}{l}\infty \\
\dot{0}\end{array}$ & ํㅜㅇ & $\hat{\sim}$ \\
\hline & \multirow{4}{*}{$\stackrel{\sim}{\circ}$} & 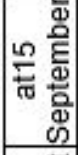 & $\stackrel{\infty}{\varrho}$ & Oे & نे & $\begin{array}{l}\mathscr{0} \\
\stackrel{0}{\circ}\end{array}$ & $\begin{array}{l}\infty \\
0 \\
0\end{array}$ & N & $\underset{\infty}{\infty}$ & $\stackrel{N}{=}$ & $\stackrel{\infty}{\sim}$ & ल & เi \\
\hline & & 㔯高 & $\stackrel{N}{\mathscr{\sigma}}$ & $\stackrel{\sim}{\sigma}$ & कृ & $\stackrel{\nabla}{\oplus}$ & $\stackrel{\circ}{\circ}$ & 우 & $\hat{N}$ & $\underset{\sigma}{\theta}$ & $\stackrel{N}{=}$ & ผn & $\underset{\text { ஸn }}{N}$ \\
\hline & & $\sum_{\pi}^{\infty} \frac{2}{3}$ & $\stackrel{\sim}{ \pm}$ & $\underset{\infty}{+}$ & مी & $\stackrel{\sim}{\sim}$ & $\hat{\infty}$ & $\hat{\omega}$ & $\stackrel{\sim}{\mathscr{\sigma}}$ & $\stackrel{n}{\sim}$ & $\stackrel{\infty}{\circ}$ & गें & $\begin{array}{l}\infty \\
1\end{array}$ \\
\hline & & 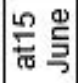 & $\stackrel{\infty}{N}$ & L & $\begin{array}{l}\infty \\
\sim \\
N\end{array}$ & $\underset{\infty}{+}$ & 요 & $\stackrel{m}{\dot{v}}$ & $\stackrel{\infty}{m}$ & $\stackrel{\sim}{\circ}$ & $\stackrel{N}{N}$ & in & $\stackrel{\omega}{\sim}$ \\
\hline & ن일 & & $N$ & $\forall$ & $\omega$ & $N$ & $\forall$ & $\omega$ & $N$ & $\nabla$ & $\omega$ & & \\
\hline & 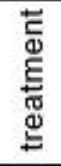 & & & & & & & & & & & $\begin{array}{l}\overline{\underline{\underline{g}}} \\
\text { 동 }\end{array}$ & 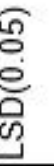 \\
\hline
\end{tabular}




\section{B. (plant oils):}

Severity of infection with the disease was also responded by the application of any tested oil (Table 2). It was decreased; in Crimson cv.; $19.2 \%$ in control plants to 4.1, 4.4 , and $5.2 \%$ in the plants treated with the highest concentration $(4.5 \mathrm{ml} / \mathrm{l})$ of castor, thyme and mint oils; respectively; at June 2015. These were $15.7 \%$ (control) and 0.5 , 2.5 and $4.5 \%$ respectively in Flame cv At September 2015 and in the same respect; severity of infection in Crimson cultivar recorded $40.8 \%$ (control) 6.7, 7.2 and 8.5\%. They were $37.8 \%$ (control) and 4.8, 5.2 and $7.7 \%$ in Flame cv.; at the same respect. However, results of 2016 showed the same effects of plant oil applications, but infection rat was higher than that of 2015 growing season.

\subsection{Effect on photosynthetic pigments:}

Application of plant extracts to grape cultivars increased chlorophyll A, B and carotenoids than control, significantly (Table 3). Total chlorophyll of both grape cultivars estimated after 3 months of leaves initiation recorded $6.35,5.95,5.70$ and $1.56 \mathrm{mg} / \mathrm{g} \mathrm{D}$ $\mathrm{W}$. in response to the individual application of castor, oleander, mint $6 \mathrm{ml} / \mathrm{land}$ nontreated control ones, respectively. At the same concentration and in the same respect; carotenoid pigments recorded 2.50 , 2.40, 2.10 and $0.59 \mathrm{mg} / \mathrm{g}$. D. W.

Data in Table (3) Chlorophyll A, Chlorophyll $B$ and carotenoids in leaf of grape $\mathrm{cv}$. increase significantly than control in response to the application of plant oils. Increasing the concentration of any tested oil up to $4.5 \mathrm{ml} / \mathrm{l}$ increased the pigments. Castor oil was more effective in the respect, followed by thyme oil. At the highest concentration, total chlorophyll was 6.90, 6.35 and $5.65 \mathrm{mg} / \mathrm{g}$ DW when castor, thyme and mint oils were individually applied, respectively. Total chlorophyll of the untreated control plants was only $1.56 \mathrm{mg} / \mathrm{g}$ DW in the same respect; carotenoids were 2.62, 2.45 and $2.26 \mathrm{mg} / \mathrm{g} \mathrm{D}$. W., for oil treatments and $0.59 \mathrm{mg} / \mathrm{g} \mathrm{D}$. W. for control plants. The ratios of total chlorophyll: the carotenoids were also increased than control, significantly.

\subsection{Effect on water relations:}

Results given in Table (4) clear that the application of plant extracts to grape cultivars increased total water content, relative water content, and sclerophylly degree than control, significantly. Mean while leaf water deficit of all treated plants showed a significant reduction compared with the un treated plants. castor and oleander extracts give the best results. Total water content in grape leaves recorded $96.72,95.34,94,65$ and $82.32 \%$, respectively with castor, oleander, $\operatorname{mint}(6 \mathrm{~g} / \mathrm{l})$ of each and control. In the same respect, relative water content was 63.84, 64.82, 65.83 and $40.8 \%$, at the same extracts concentration.

In the same respect, relative water content was $63.84,64.82,65.83$ and $40.8 \%$, at the same extracts concentration. In the same table Total water content and relative water significantly higher than control, in both grape cultivars, treated with different plant oils there were no significant variations between the tested oils and/ or their used concentration. On the other hand, the percentage of leaf water deficit of control plants was significantly higer than those plants treated with oils.

\subsection{Effect on yield components and production:}

Results presented in Table (5) clear that the application of plant extracts increased yield components of Crimson cV. Than control significantly; both at 2015 and 2016 season. The average number of cluster/plant was increased from 15 in control treatment to 27,27 and 28 when the extracts of castor, mint and oleander $(6 \mathrm{ml} / \mathrm{l})$ were individually applied at 2015. These numbers were 26, 25 and 25 clusters per plant at 2016, respectively and those of control were only 18/plant. The average length of cluster was also increased than control in response to 


\begin{tabular}{|c|c|c|c|c|c|c|c|c|c|c|c|c|c|}
\hline \multirow{8}{*}{$\begin{array}{l}\text { 음 } \\
\text { 员 } \\
\text { 号 }\end{array}$} & \multirow{4}{*}{$\stackrel{0}{\circ}$} & 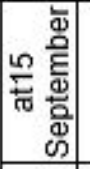 & $\underset{N}{\simeq}$ & $\stackrel{m}{=}$ & क) & $\stackrel{0}{\sim}$ & $\stackrel{\text { ָे }}{\sim}$ & r & $\stackrel{\sigma}{\sim}$ & $\stackrel{\infty}{\underset{+}{+}}$ & $\stackrel{m}{\sigma}$ & 每 & กั \\
\hline & & 览 & 竎 & $\stackrel{\varphi}{\circ}$ & $\underset{\omega}{\tilde{c}}$ & $\stackrel{\infty}{\stackrel{\infty}{\sim}}$ & $\stackrel{\infty}{\leftarrow}$ & $\begin{array}{l}\infty \\
\omega\end{array}$ & $\begin{array}{l}\infty \\
\stackrel{\infty}{\leftarrow}\end{array}$ & $\stackrel{\sim}{m}$ & 穴 & 웅 & ले \\
\hline & & $\frac{2}{\pi} \frac{2}{3}$ & $\underset{ָ}{ָ}$ & 웅 & No & $\stackrel{\infty}{m}$ & $\stackrel{\Upsilon}{=}$ & ๗ֶ & $\stackrel{\varphi}{\square}$ & $\stackrel{N}{\sim}$ & N & m & m \\
\hline & & 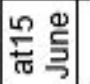 & 웅 & O & 웅 & $\stackrel{ }{\circ}$ & $\begin{array}{l}\infty \\
\infty\end{array}$ & $\stackrel{4}{L}$ & $\stackrel{\infty}{\stackrel{\infty}{\sim}}$ & ํ. & $\underset{0}{\forall}$ & ํํ & N \\
\hline & \multirow{4}{*}{$\stackrel{\sim}{\circ}$} & 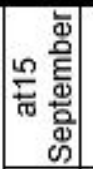 & $\begin{array}{l}\stackrel{0}{N} \\
\stackrel{\sim}{r}\end{array}$ & $\begin{array}{l}\sigma \\
\sigma\end{array}$ & 'ó & $\stackrel{N}{m}$ & ํㅜㅇ & N & $\underset{N}{N}$ & $\begin{array}{l}\infty \\
\stackrel{\infty}{\Gamma}\end{array}$ & L & $\begin{array}{l}\infty \\
0 \\
0\end{array}$ & $\stackrel{\varphi}{m}$ \\
\hline & & 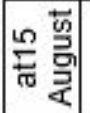 & $\stackrel{\infty}{\leftarrow}$ & ஸ̃ & 寸ै & $\stackrel{\sigma}{\sim}$ & $\begin{array}{l}\infty \\
\sigma^{\prime}\end{array}$ & $\begin{array}{l}\infty \\
\omega \\
0\end{array}$ & $\stackrel{\oplus}{\oplus}$ & $\stackrel{N}{\check{N}}$ & $M$ & $\begin{array}{l}m \\
\infty \\
m\end{array}$ & की \\
\hline & & 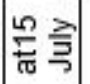 & 뭉 & $m$ & $\stackrel{\varphi}{\omega}$ & $\stackrel{\infty}{\leftarrow}$ & $\infty$ & बr & $\stackrel{L}{\dot{I}}$ & $\mp$ & ๑ & $\begin{array}{l}\text { Ln } \\
\text { Ln }\end{array}$ & $\begin{array}{l}\infty \\
\text { m }\end{array}$ \\
\hline & & 兄 & L & $\stackrel{0}{N}$ & 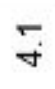 & $\stackrel{N}{\sigma}$ & N & 寸 & $\stackrel{\infty}{\circ}$ & $\stackrel{\infty}{\infty}$ & นั & $\begin{array}{l}N \\
\sigma \\
\sigma\end{array}$ & $\stackrel{\circ}{\text { N }}$ \\
\hline \multirow{8}{*}{$\begin{array}{l}\stackrel{\varpi}{E} \\
\frac{\pi}{\amalg}\end{array}$} & \multirow{4}{*}{$\frac{\infty}{\circ}$} & 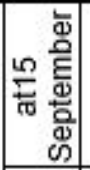 & $\stackrel{\varphi}{\dot{U}}$ & $\begin{array}{l}\omega \\
\infty\end{array}$ & क) & $\stackrel{g}{\dot{I}}$ & $\tilde{\sigma}$ & $\stackrel{N}{N}$ & $\stackrel{\oplus}{\oplus}$ & $\begin{array}{l}\text { 웅 } \\
0\end{array}$ & $\begin{array}{l}\text { L } \\
\infty\end{array}$ & $\begin{array}{l}N \\
\text { m }\end{array}$ & $m$ \\
\hline & & 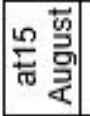 & $\stackrel{\infty}{m}$ & $\hat{N}$ & $\underset{\omega}{0}$ & $\stackrel{\infty}{m}$ & $\begin{array}{l}\forall \\
\infty\end{array}$ & 寸ै & $\stackrel{N}{n}$ & ำ & $\hat{N}$ & ๗̃. & "n \\
\hline & & 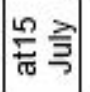 & $\stackrel{\infty}{\stackrel{\infty}{\sim}}$ & क & $\stackrel{\sigma}{\forall}$ & $\stackrel{\sigma}{\sim}$ & $N$ & ָั & $\underset{v}{\stackrel{v}{Z}}$ & ণ & $\begin{array}{l}\infty \\
\omega\end{array}$ & นn & $\stackrel{\varphi}{m}$ \\
\hline & & 旡 & $\hat{\infty}$ & ச & $\stackrel{\infty}{m}$ & 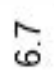 & ?ִ & $\stackrel{\sim}{\forall}$ & की & $m$ & $\underset{\omega}{\sim}$ & ํㅜㄷ & $\stackrel{+}{\sim}$ \\
\hline & \multirow{4}{*}{$\stackrel{n}{\circ}$} & 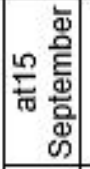 & $\stackrel{\square}{=}$ & $\underset{\infty}{\infty}$ & $\stackrel{\infty}{+}$ & $\stackrel{\circ}{\stackrel{0}{\sigma}}$ & $\begin{array}{l}9 \\
\infty\end{array}$ & กี & $\stackrel{5}{n}$ & $\stackrel{m}{\sigma}$ & $\hat{N}$ & m & की \\
\hline & & 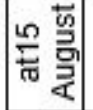 & $\stackrel{\infty}{0}$ & $\stackrel{0}{N}$ & $\stackrel{m}{\forall}$ & $\stackrel{+}{=}$ & $\underset{\infty}{\sim}$ & $\stackrel{\infty}{\dot{\gamma}}$ & ले & $N_{\infty}^{\prime}$ & $\underset{0}{~}$ & मn & $\stackrel{\omega}{m}$ \\
\hline & & $\sum_{\pi}^{\infty}$ & ब) & 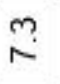 & $\stackrel{\infty}{\sim}$ & $\stackrel{N}{0}$ & N & $\stackrel{\nabla}{\forall}$ & 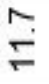 & $\stackrel{\infty}{N}$ & Li & लें & $\begin{array}{l}\infty \\
m\end{array}$ \\
\hline & & 占 & ڤึ & L & ? & क) & $\underset{\omega}{~}$ & $\stackrel{\sim}{\sim}$ & M & சै & $\stackrel{\circ}{\forall}$ & L & $\stackrel{\infty}{N}$ \\
\hline & 응 & & $N$ & $\stackrel{m}{m}$ & $\stackrel{4}{+}$ & $N$ & $\stackrel{m}{m}$ & $\stackrel{4}{8}$ & $N$ & m & $\stackrel{L}{\forall}$ & & (I) \\
\hline & 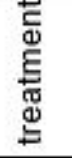 & & & 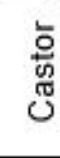 & & & $\stackrel{E}{E}$ & & & 謍 & & 氖 & $\begin{array}{l}0 \\
0 \\
\text { vi } \\
i\end{array}$ \\
\hline
\end{tabular}


M. M. Ammar, et al.,

\begin{tabular}{|c|c|c|c|c|c|c|c|c|c|c|c|c|}
\hline \multirow{4}{*}{$\begin{array}{l}\frac{\omega}{\bar{D}} \\
\frac{\bar{L}}{\bar{E}} \\
\frac{\pi}{\square}\end{array}$} & 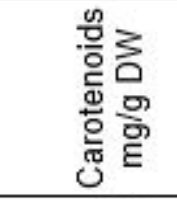 & 워- & స్ & ָู & $\stackrel{m}{m}$ & 은 & $\stackrel{\text { fo }}{\mathrm{v}}$ & $\stackrel{\text { Iొ }}{-}$ & $\stackrel{\circ}{i}$ & 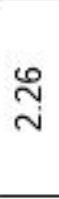 & $\begin{array}{l}\text { 。 } \\
0 \\
0\end{array}$ & $\stackrel{\sim}{0}$ \\
\hline & 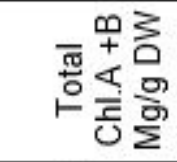 & $\begin{array}{l}\text { ळ } \\
\text { m. }\end{array}$ & के & क) & $\begin{array}{l}\text { @ } \\
\text { ले }\end{array}$ & 买 & लn & $\begin{array}{l}\text { مొ } \\
\text { లై }\end{array}$ & $\begin{array}{l}\text { g } \\
\dot{\forall}\end{array}$ & $\begin{array}{l}\text { 岇 } \\
\text { ద }\end{array}$ & $\stackrel{6}{\circ}$ & 웅 \\
\hline & 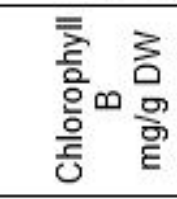 & $\stackrel{\text { రె }}{-}$ & $\stackrel{\substack{\llcorner \\
\sim}}{\mathrm{N}}$ & ๙ั & $\stackrel{\circ}{\longrightarrow}$ & 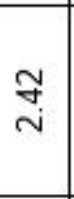 & 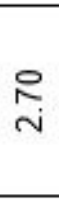 & $\stackrel{\text { fo }}{-}$ & 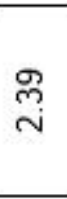 & 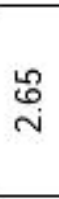 & छ़ & $\stackrel{2}{\circ}$ \\
\hline & 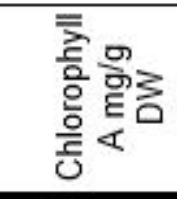 & స్ & $\stackrel{\text { ॄे }}{\text { mे }}$ & $\stackrel{\circ}{\forall}$ & $\stackrel{\infty}{i}$ & 이 & 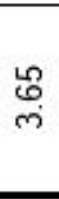 & $\bar{i}$ & 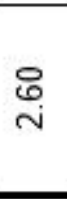 & 우 & శึ & $\begin{array}{l}\text { Jे } \\
\stackrel{0}{\circ}\end{array}$ \\
\hline & 돟 & $\sim$ & $\stackrel{\sim}{m}$ & $\stackrel{L}{\forall}$ & $\sim$ & $\stackrel{\sim}{m}$ & $\stackrel{4}{\forall}$ & $\sim$ & $\stackrel{\sim}{m}$ & $\stackrel{4}{\forall}$ & & \\
\hline & 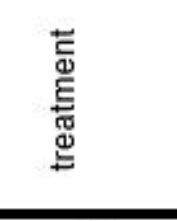 & & 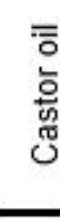 & & & $\begin{array}{l}\overline{\bar{O}} \\
\text { ఏ } \\
\text { E} \\
\text { ह }\end{array}$ & & & $\begin{array}{l}\overline{\bar{o}} \\
\overline{\underline{E}}\end{array}$ & & $\begin{array}{l}\overline{0} \\
\text { 음 } \\
\end{array}$ & 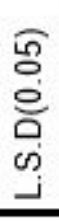 \\
\hline \multirow{4}{*}{ 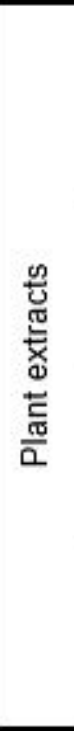 } & 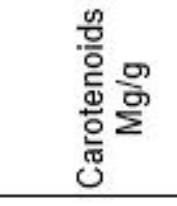 & $\stackrel{\text { mे }}{-}$ & 은 & ํㅠ & ్ㅜ & ֻे & $\stackrel{\text { 巳 }}{\mathrm{i}}$ & $\stackrel{\stackrel{L}{N}}{=}$ & $\stackrel{\text { 욤 }}{-}$ & 은 & $\begin{array}{l}\text { م } \\
0 \\
0\end{array}$ & $\stackrel{\sim}{0}$ \\
\hline & 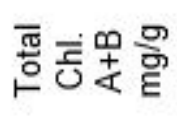 & రె & $\stackrel{\text { ᄋ }}{+}$ & 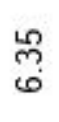 & ले & $\stackrel{\stackrel{\llcorner}{\circ}}{\forall}$ & $\begin{array}{l}\text { ผn } \\
\text { เn }\end{array}$ & $\stackrel{\text { Nִ }}{\text { mె }}$ & $\stackrel{\stackrel{\sim}{N}}{\forall}$ & $\hat{\text { ș }}$ & $\stackrel{\mathscr{L}}{\longrightarrow}$ & 。․ \\
\hline & 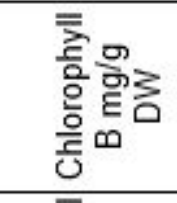 & 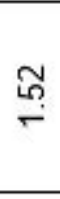 & $\stackrel{\circ}{\sim}$ & $\stackrel{\circ}{\sim}$ & $\stackrel{\text { ㅁ }}{\longrightarrow}$ & $\stackrel{\text { ڤn }}{-}$ & $\stackrel{\text { ㅁ }}{\text { i }}$ & $\stackrel{\text { m }}{\longrightarrow}$ & $\stackrel{\circ}{-}$ & ֻొ & छ़ & ¿̊. \\
\hline & 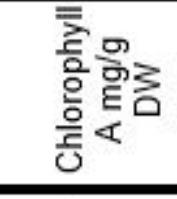 & 음 & ్. & $\begin{array}{l}\stackrel{2}{\infty} \\
\text { m. }\end{array}$ & $\stackrel{\circ}{\mathrm{i}}$ & $\begin{array}{l}\stackrel{0}{6} \\
\text { i }\end{array}$ & $\begin{array}{l}\stackrel{L}{\omega} \\
\text { m. }\end{array}$ & ค् & $\stackrel{\text { 尔 }}{\mathrm{N}}$ & o & ๙ั & $\stackrel{2}{\circ}$ \\
\hline & ठํ & $N$ & $\nabla$ & $\omega$ & $N$ & $\nabla$ & $\omega$ & $N$ & $\nabla$ & $\omega$ & & \\
\hline & 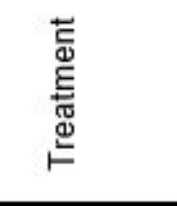 & & & & & 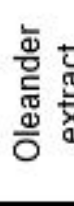 & & & 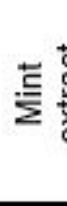 & & 올 & 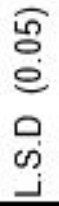 \\
\hline
\end{tabular}




\begin{tabular}{|c|c|c|c|c|c|c|c|c|c|c|c|c|}
\hline \multirow{4}{*}{ 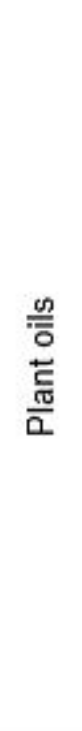 } & 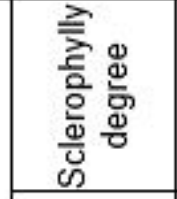 & 先 & $\begin{array}{l}\stackrel{\circ}{\circ} \\
\text { హे }\end{array}$ & $\begin{array}{l}\bar{\infty} \\
\text { ळू }\end{array}$ & $\begin{array}{l}\hat{\sigma} \\
\text { ڤू }\end{array}$ & $\begin{array}{l}\text { ळ } \\
\text { డి }\end{array}$ & $\begin{array}{l}\text { के } \\
\text { హे }\end{array}$ & $\begin{array}{l}\text { जै } \\
\text { ले }\end{array}$ & $\begin{array}{l}\text { హิ } \\
\text { ผू }\end{array}$ & $\begin{array}{l}8 \\
\text { ผू }\end{array}$ & $\begin{array}{l}\text { m } \\
\infty \\
\infty\end{array}$ & ๙ู \\
\hline & 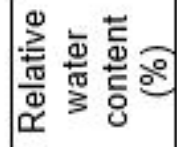 & $\begin{array}{l}\widehat{\omega} \\
\text { ம் }\end{array}$ & $\begin{array}{l}\text { ले } \\
\text { छै }\end{array}$ & $\begin{array}{l}\infty \\
\infty \\
\tilde{\omega}\end{array}$ & $\begin{array}{l}\frac{L}{2} \\
\stackrel{6}{6}\end{array}$ & $\begin{array}{l}\text { : } \\
\text { రి }\end{array}$ & 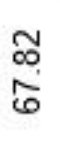 & ָூ & กુ & $\begin{array}{l}-\infty \\
\dot{0} \\
\dot{\sigma}\end{array}$ & $\begin{array}{l}\infty \\
\text { Oे }\end{array}$ & নু \\
\hline & 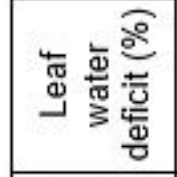 & $\begin{array}{l}\bar{y} \\
\text { ले }\end{array}$ & 先 & $\begin{array}{l}\infty \\
\infty \\
m\end{array}$ & $\begin{array}{l}\frac{\pi}{2} \\
\text { Ni }\end{array}$ & స్ & $\begin{array}{l}\bar{\sigma} \\
\infty \\
\sim\end{array}$ & $\begin{array}{l}\text { กี } \\
\text { กี }\end{array}$ & ¿ & 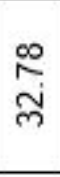 & 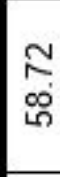 & 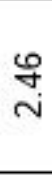 \\
\hline & 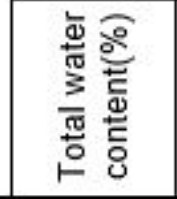 & ్ㅗ & $\begin{array}{l}\text { चे } \\
\text { Цू' }\end{array}$ & $\begin{array}{l}\text { N } \\
\mathscr{6}\end{array}$ & $\begin{array}{l}\text { ळ } \\
\text { ठే }\end{array}$ & $\begin{array}{l}\bar{N} \\
\text { హे }\end{array}$ & $\begin{array}{l}\text { ले } \\
\text { హें }\end{array}$ & กี & $\begin{array}{l}\text { ळ } \\
\text { Бे }\end{array}$ & ๙ึ. & กี & $\stackrel{m}{\sim}$ \\
\hline \multicolumn{2}{|c|}{ ¿ू. } & $N$ & $\stackrel{n}{m}$ & $\stackrel{n}{\forall}$ & $\sim$ & $\stackrel{\mathrm{m}}{\mathrm{m}}$ & $\stackrel{\sim}{\forall}$ & $N$ & $\stackrel{n}{m}$ & $\stackrel{\sim}{\forall}$ & & \multirow[b]{2}{*}{$\begin{array}{l}\hat{2} \\
\dot{0} \\
e \\
0 \\
0 \\
\text { on }\end{array}$} \\
\hline \multicolumn{2}{|r|}{ 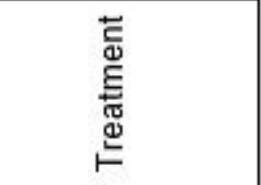 } & \multicolumn{3}{|c|}{ 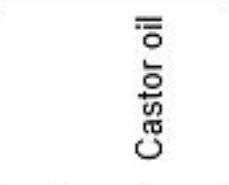 } & \multicolumn{3}{|c|}{$\begin{array}{l}\overline{\bar{o}} \\
\stackrel{\searrow}{E} \\
\text { હ્ }\end{array}$} & \multicolumn{3}{|c|}{$\begin{array}{l}\overline{\bar{o}} \\
\stackrel{\underline{\underline{E}}}{\Sigma}\end{array}$} & $\begin{array}{l}\overline{0} \\
\text { 을 } \\
\text { 등 }\end{array}$ & \\
\hline \multirow{4}{*}{ 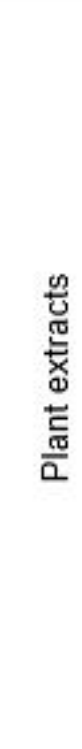 } & 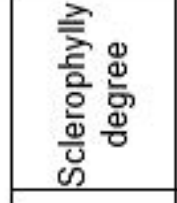 & $\begin{array}{l}\text { 巳े } \\
\text { बें }\end{array}$ & $\begin{array}{l}\mathscr{L} \\
\text { ڤึ }\end{array}$ & $\begin{array}{l}\text { क } \\
\text { ठें }\end{array}$ & $\begin{array}{l}\qquad 0 \\
\text { ठే }\end{array}$ & $\begin{array}{l}\text { ळ } \\
\text { Цू }\end{array}$ & $\begin{array}{l}\text { के } \\
\text { ผ่ }\end{array}$ & 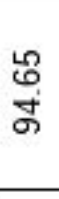 & $\begin{array}{l}\text { ूे } \\
\text { ผू }\end{array}$ & $\begin{array}{l}\infty \\
\Xi \\
\dot{\sigma}\end{array}$ & $\begin{array}{l}\text { m } \\
\infty \\
\infty\end{array}$ & รู \\
\hline & 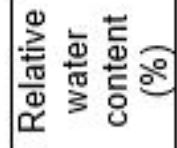 & $\begin{array}{c}\infty \\
\tilde{\omega}\end{array}$ & นึ. & $\begin{array}{l}\text { D. } \\
\text { లై }\end{array}$ & $\begin{array}{l}\stackrel{n}{R} \\
\stackrel{6}{6}\end{array}$ & $\bar{m}$ & 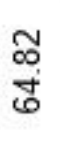 & लె & $\begin{array}{l}\stackrel{2}{2} \\
\stackrel{5}{6}\end{array}$ & $\begin{array}{l}m \\
\infty \\
\omega \\
\omega\end{array}$ & $\begin{array}{l}\infty \\
\stackrel{+}{q}\end{array}$ & $\begin{array}{l}\text { ন } \\
\text { ㄱ }\end{array}$ \\
\hline & 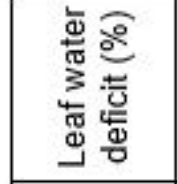 & $\begin{array}{l}\tilde{N} \\
\text { एँ } \\
\dot{m}\end{array}$ & ঙ̊? & $\begin{array}{l}\mathscr{L} \\
\stackrel{m}{ }\end{array}$ & $\begin{array}{l}\stackrel{0}{*} \\
\stackrel{\sim}{\sim}\end{array}$ & $\begin{array}{l}\text { ले } \\
\text { ळें }\end{array}$ & $\begin{array}{l}\bar{\sigma} \\
\text { बें }\end{array}$ & $\begin{array}{l}\text { बे } \\
\text { ले }\end{array}$ & $\begin{array}{l}\mathscr{N} \\
\text { ले }\end{array}$ & $\begin{array}{l}\frac{R}{2} \\
\text { m }\end{array}$ & $\underset{\infty}{N}$ & $\begin{array}{l}0 \\
\text { j }\end{array}$ \\
\hline & 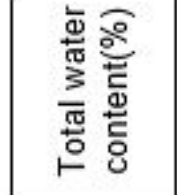 & $\begin{array}{l}m \\
\infty \\
\stackrel{\sigma}{\sigma}\end{array}$ & 守 & $\underset{\mathscr{\sigma}}{\stackrel{N}{6}}$ & $\begin{array}{l}\text { ○ } \\
\dot{\sigma}\end{array}$ & ஸे & $\begin{array}{l}\text { ले } \\
\text { ळூ }\end{array}$ & $\begin{array}{l}\text { Q } \\
\text { هั }\end{array}$ & 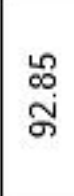 & $\begin{array}{l}\text { ڤ̊ } \\
\text { హ் }\end{array}$ & ก̃ & $\stackrel{m}{\forall}$ \\
\hline \multicolumn{2}{|c|}{ ठั } & $N$ & $\nabla$ & $\omega$ & $N$ & $\forall$ & 6 & $N$ & $\nabla$ & $\omega$ & & \\
\hline \multicolumn{2}{|r|}{ 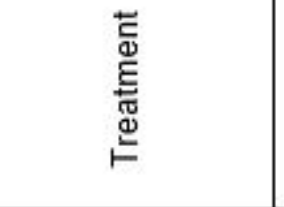 } & \multicolumn{3}{|c|}{ 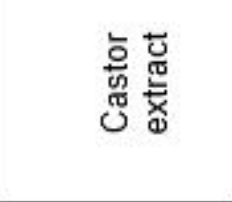 } & \multicolumn{3}{|c|}{ 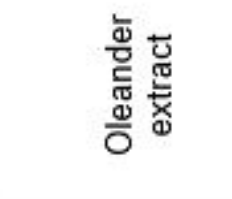 } & \multicolumn{3}{|c|}{ 志莺 } & 产 & e \\
\hline
\end{tabular}




\begin{tabular}{|c|c|c|c|c|c|c|c|c|c|c|c|c|c|}
\hline \multirow{6}{*}{ 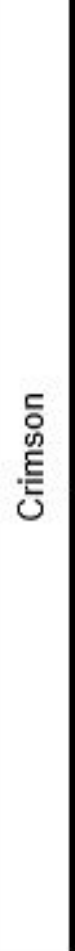 } & \multirow{3}{*}{$\stackrel{\circ}{\sim}$} & 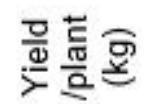 & ò & $\hat{\circ}$ & $\stackrel{\text { L }}{=}$ & $\underset{\infty}{N}$ & $\stackrel{\circ}{\circ}$ & $\stackrel{\simeq}{=}$ & $\begin{array}{l}\circ \\
\infty\end{array}$ & ஸ̆ & $\stackrel{m}{\circ}$ & 오 & $\stackrel{\text { a }}{\mathrm{v}}$ \\
\hline & & $\begin{array}{l}\text { 总䓌 } \\
\text { 总总 }\end{array}$ & 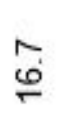 & $\stackrel{0}{\cong}$ & $\begin{array}{l}\sigma \\
\oplus\end{array}$ & $\begin{array}{l}0 \\
\varrho \\
\varrho\end{array}$ & $\stackrel{N}{\approx}$ & $\stackrel{m}{=}$ & $\stackrel{3}{\mathscr{\sigma}}$ & $\stackrel{0}{=}$ & $\stackrel{ \pm}{=}$ & $\stackrel{m}{ \pm}$ & $\stackrel{\infty}{\sim}$ \\
\hline & & 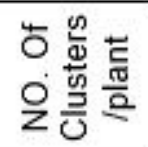 & 옴 & 올 & 完 & $\stackrel{\text { ㅇ }}{\circ}$ & $\stackrel{\circ}{\sim}$ & 옹 & $\begin{array}{l}\stackrel{\circ}{\circ} \\
\stackrel{2}{2}\end{array}$ & ํㅗ & ì & 옴 & ले \\
\hline & \multirow{3}{*}{$\stackrel{\text { Ln }}{\sim}$} & 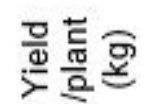 & \%ั & 뭉 & $\stackrel{\sigma}{=}$ & $\begin{array}{l}L_{\infty}^{\infty} \\
\infty\end{array}$ & ָ̃ & $\stackrel{n}{=}$ & $\stackrel{\sigma}{r}$ & Oे & 웅 & $\stackrel{\varphi}{\omega}$ & $\stackrel{\varphi}{\sim}$ \\
\hline & & 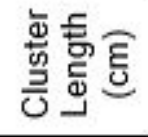 & $\stackrel{N}{\sim}$ & $\stackrel{?}{\sim}$ & $\stackrel{m}{\sim}$ & $\stackrel{\infty}{\check{1}}$ & $\stackrel{\infty}{\stackrel{\infty}{=}}$ & $\stackrel{m}{\infty}$ & $\begin{array}{l}\sigma \\
6\end{array}$ & $\stackrel{m}{m} \underset{\infty}{\infty}$ & $\stackrel{0}{\circ}$ & $\stackrel{\circ}{\dot{j}}$ & $\stackrel{\llcorner}{\sim}$ \\
\hline & & 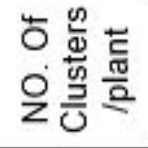 & $\frac{0}{i}$ & ๗ે & $\begin{array}{l}\text { Dे } \\
\text { ì }\end{array}$ & $\stackrel{\circ}{=}$ & 오 & 鬲 & $\stackrel{0}{\check{2}}$ & 옴 & 올 & $\begin{array}{l}0 \\
\infty \\
-\infty\end{array}$ & $\stackrel{\vec{m}}{\mathrm{~m}}$ \\
\hline \multirow{6}{*}{$\begin{array}{l}\stackrel{\oplus}{E} \\
\stackrel{\pi}{\amalg} \\
\end{array}$} & \multirow{3}{*}{$\stackrel{\oplus}{\circ}$} & 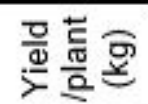 & $\stackrel{\sim}{\sigma}$ & $\stackrel{\infty}{\circ}$ & $\stackrel{\sigma}{=}$ & $\begin{array}{l}L \\
\infty \\
\infty\end{array}$ & $\stackrel{N}{\circ}$ & $\stackrel{+}{=}$ & $\underset{\infty}{m}$ & बें & 훙 & $\begin{array}{l}n \\
6 \\
6\end{array}$ & $\stackrel{\sim}{m}$ \\
\hline & & 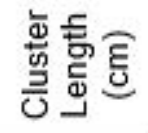 & $\stackrel{N}{\kappa}$ & $\stackrel{N}{=}$ & $\begin{array}{l}\mathscr{0} \\
\stackrel{0}{\circ}\end{array}$ & $\begin{array}{l}\mathscr{\sigma} \\
6\end{array}$ & $\stackrel{m}{F}$ & $\begin{array}{c}m \\
\infty\end{array}$ & $\stackrel{m}{\sim}$ & $\stackrel{m}{=}$ & $\stackrel{0}{\sim}$ & 웅 & $\stackrel{\llcorner}{\sim}$ \\
\hline & & 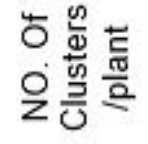 & $\stackrel{\circ}{\frac{1}{N}}$ & 号 & 옹 & 옴 & 옹 & 옴 & 옴 & $\stackrel{\circ}{\grave{N}}$ & 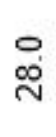 & $\begin{array}{l}0 \\
\text { i }\end{array}$ & $\stackrel{\infty}{\sim}$ \\
\hline & \multirow{3}{*}{ 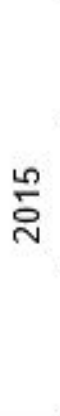 } & 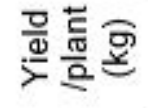 & $\stackrel{\varphi}{\sigma}$ & $\stackrel{0}{\circ}$ & $\stackrel{\circ}{\sim}$ & $\underset{\infty}{\infty}$ & के & $\stackrel{+}{=}$ & $\stackrel{n}{N}$ & ஸे & $\stackrel{\circ}{\circ}$ & की & m \\
\hline & & 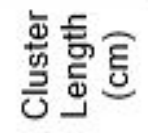 & $\begin{array}{l}\stackrel{\sigma}{0} \\
\stackrel{6}{\circ}\end{array}$ & $\stackrel{\circ}{\Gamma}$ & $\stackrel{\varphi}{\stackrel{0}{\sim}}$ & $\stackrel{N}{\sim}$ & $\begin{array}{l}\stackrel{5}{ } \\
6\end{array}$ & $\begin{array}{l}\circ \\
\stackrel{0}{\circ} \\
\stackrel{0}{2}\end{array}$ & $\underset{\sim}{\stackrel{N}{\sim}}$ & $\begin{array}{l}\circ \\
\stackrel{\infty}{\leftarrow}\end{array}$ & $\stackrel{\text { ? }}{\stackrel{2}{\sim}}$ & $\stackrel{\varphi}{\dot{J}}$ & $\stackrel{\forall}{\sim}$ \\
\hline & & 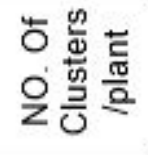 & $\stackrel{ }{N}$ & $\stackrel{\circ}{\sim}$ & $\stackrel{0}{\text { N }}$ & $\stackrel{\circ}{\infty}$ & $\stackrel{\circ}{\stackrel{N}{N}}$ & $\stackrel{\circ}{\text { ஸे }}$ & $\begin{array}{l}\circ \\
\stackrel{\infty}{-}\end{array}$ & 옴 & $\begin{array}{l}0 \\
\dot{v}\end{array}$ & 웅 & N \\
\hline & Еั & हे & $N$ & $\tilde{m}$ & $\stackrel{8}{7}$ & $N$ & $\tilde{m}$ & $\stackrel{0}{+}$ & $N$ & $\stackrel{n}{n}$ & $\stackrel{5}{+}$ & & \\
\hline \multicolumn{3}{|c|}{ 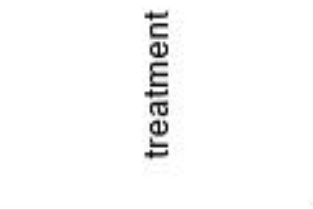 } & \multicolumn{3}{|c|}{ 前 } & \multicolumn{3}{|c|}{ 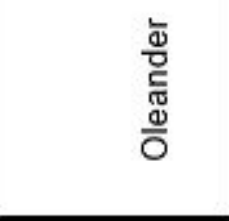 } & \multicolumn{3}{|c|}{ 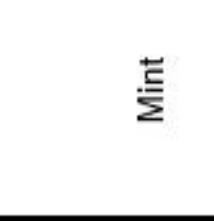 } & & 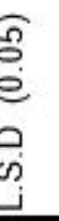 \\
\hline
\end{tabular}


the application of plant extracts. A significant incrsement was observed in most cases. However; highly significant increase was observed in yield and its components of the treated plants compared to the untreated control ones. At 2015 season ; control plants yielded an average of $5.6 \mathrm{~kg}$ While the treated plants with $6 \mathrm{ml} / \mathrm{l}$ of either castor, oleander and mint produced the average of $11.9,11.5$ and $10.7 \mathrm{~kg}$;respectively. These were 11.5, 11.2 and 10.3 at 2016 season; respectively; when control plants produced an average of $5 \mathrm{~kg}$ only. However; results of yield and its components of Flame cultivar at 2015 and 2016; as affected by the application of plant water extracts; are given in grape table. The average number of clusters/plant was increased than control at 2015 season; significantly; in most cases. The same observations were noticed for cluster length and yield and its components. At 2016 growing season; significant increments were noticed between the treated and nontreated plants in both average cluster length and the amount of yield /plant. Average number of clusters /plant was insignificantly increased than control; in response to the application of extracts. Generally; increasing the concentration of any tested extract resulted in more yield and its components, but insignificantly.

Results of yield and its components of Crimson grape cultivar both at 2015 and 2016 seasons were present in Table (6) These results clear that the application of plant oils to grape plants gave very high significant increase in yield components in comparison with control . Castor oil (3.5 and lor $4.5 \mathrm{ml} / \mathrm{l}$ ) showed the best results in all yield components both at 2015 and 2016 seasons. Yield of control plants was $5.6 \mathrm{~kg}$. /plant at 2015. However; the average yield/plant reached 10.9 and $11.8 \mathrm{~kg}$ when castor oil was applied at 3.5 and $4.5 \mathrm{ml} / \mathrm{l}$ respectively. In the same respect; these were 10.2 and $11.3 \mathrm{~kg} / \mathrm{plant}$; at 2016; when control plants yielded an average of 5 $\mathrm{kg} /$ plant only. As for Flame cultivar Table (6) castor and thyme oils gave the best positive effects on yield production at both years of investigation. In general; all applications showed significant increase in yield components of both grape cultivars. In the meantime ; increasing the concentration of any tested oil increased yield production ; but insignificantly in most cases.

\section{Discussion:}

Results of the present investigation clear that, the individual application of either plant water extracts or plant oils showed significant reduction in grape downy mildew disease incidence. The reduction in downy mildew disease refer to, the effect of chemical composition of the plant oils and extracts and which effect that on the structure of epidermis cells and stomatal opening and closer. This reason clearly effect on the germination of fungi spores and the growth of fungi mycellium. Castor plant extract gave the best results both at 2015 and 2016. For Crimson and Flame cultivars increasing the concentration of any tested extract and lor oil showed significant reduction of the disease incidence. However, similar results were observed by Achimu and Schlosser (1992) who attributed the antifungal properties of the extract to the inhibition of the direct germination of sporangia. Klinkenberg et al., (1998) indicated that induced resistance can be an additional tool in the integrated control of disease of diseases caused by Oomycetes. Cohen et al., (2002) and (2006) mentioned that extracts of Inula visicosa have at least seven fungicidal compounds. Scanna vini et al., (2004) revealed that plant extracts stimulate grape plant development and natural disease resistance

Kast and Bleyer (2006) found that the antifungal effect, of plant extracts, increased as the extract concentration increased. Rajeswari et al., (2008) revealed that the neem seed kernel extract at 5\%, significantly inhibited the sporangial germination (75.36\%) of p.viticola and effectively inhibited the disease development Gindro 


\begin{tabular}{|c|c|c|c|c|c|c|c|c|c|c|c|c|c|}
\hline \multirow{6}{*}{ 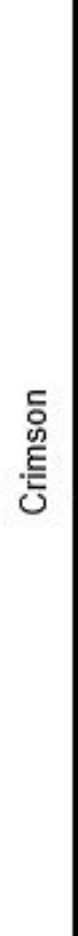 } & \multirow{3}{*}{$\frac{\varphi}{\sim}$} & 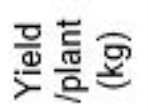 & $\stackrel{\sim}{\sigma}$ & $\stackrel{\circ}{\circ}$ & $\stackrel{\infty}{=}$ & 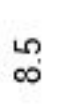 & $\stackrel{N}{0}$ & $\stackrel{m}{=}$ & $\bar{\infty}$ & के & 숭 & 오 & $\stackrel{2}{1}$ \\
\hline & & 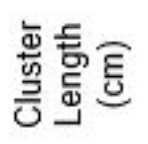 & $\stackrel{\circ}{\infty}$ & $\stackrel{n}{\infty}$ & 웅 & $\underset{\infty}{\infty}$ & $\stackrel{\stackrel{\llcorner}{\sigma}}{\stackrel{\sigma}{\Gamma}}$ & $\stackrel{m}{\sigma}$ & $\stackrel{+}{\infty}$ & $\stackrel{\sim}{\sigma}$ & $\stackrel{+}{\infty}$ & $\stackrel{m}{ \pm}$ & $\stackrel{\infty}{\sim}$ \\
\hline & & 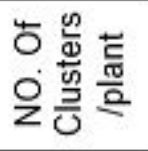 & ํ. & 옴 & ले & 옹 & 完 & 옴 & $\begin{array}{l}\circ \\
\stackrel{1}{\leftarrow}\end{array}$ & $\frac{0}{\bar{N}}$ & 品 & 옴 & $\underset{N}{N}$ \\
\hline & \multirow{3}{*}{ 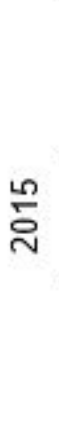 } & 흥 壳 & $\underset{\infty}{\sigma}$ & $\hat{\circ}$ & $\stackrel{\sigma}{\leftarrow}$ & $\underset{\infty}{\sim}$ & 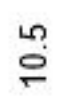 & $\stackrel{\circ}{\circ}$ & $\underset{\infty}{\forall}$ & $\tilde{a}$ & $\stackrel{n}{\circ}$ & $\stackrel{\omega}{\omega}$ & $\stackrel{\sim}{m}$ \\
\hline & & 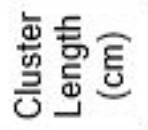 & $\stackrel{\sigma}{\sim}$ & $\underset{\infty}{\infty}$ & $\begin{array}{l}\ddot{2} \\
\leftarrow\end{array}$ & $\stackrel{+}{\infty}$ & $\stackrel{\infty}{\stackrel{\infty}{\Gamma}}$ & $\begin{array}{l}\stackrel{\sigma}{\infty} \\
\infty\end{array}$ & $\stackrel{m}{\sigma}$ & $\begin{array}{l}\stackrel{\leftrightarrow}{\infty} \\
\stackrel{\infty}{\leftarrow}\end{array}$ & $\stackrel{\sim}{\sigma}$ & $\stackrel{\circ}{\stackrel{0}{+}}$ & $\stackrel{m}{\sim}$ \\
\hline & & 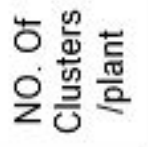 & ஸें & $\begin{array}{l}0 \\
\text { i }\end{array}$ & ํㅗ․ & $\stackrel{\circ}{\circ}$ & $\stackrel{\circ}{\sim}$ & $\begin{array}{l}\text { i } \\
\text { ì }\end{array}$ & $\stackrel{\circ}{\circ}$ & $\stackrel{\text { N }}{\text { N }}$ & ले & $\begin{array}{l}\circ \\
\infty\end{array}$ & $\stackrel{+}{\sim}$ \\
\hline \multirow{6}{*}{$\begin{array}{l}\stackrel{Ð}{E} \\
\frac{\pi}{\amalg}\end{array}$} & \multirow{3}{*}{$\stackrel{n}{\sim}$} & 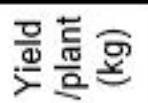 & $\stackrel{\infty}{\sigma}$ & $\hat{\circ}$ & $\stackrel{9}{=}$ & $m$ & 웅 & $\stackrel{\circ}{\circ}$ & $\stackrel{?}{r}$ & $m$ & $\stackrel{N}{\circ}$ & ए? & $\stackrel{\operatorname{Ln}}{\sim}$ \\
\hline & & 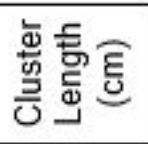 & $\underset{\infty}{\stackrel{+}{\infty}}$ & $\underset{\infty}{\stackrel{\infty}{\infty}}$ & 足 & $\stackrel{m}{\infty}$ & 함 & $\stackrel{\sigma}{\sigma}$ & $\underset{\infty}{\infty}$ & $\stackrel{\stackrel{\sim}{\sigma}}{\stackrel{\sigma}{\leftarrow}}$ & $\begin{array}{l}\underset{\infty}{\sigma} \\
\infty\end{array}$ & 옹 & $\stackrel{\infty}{-}$ \\
\hline & & 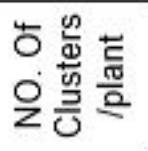 & $\stackrel{\circ}{\frac{1}{N}}$ & $\begin{array}{l}0 \\
\ddot{v}\end{array}$ & ำ & ले & $\stackrel{\circ}{\text { N }}$ & 吕 & $\stackrel{\circ}{\circ}$ & $\stackrel{\circ}{\tilde{N}}$ & 옹 & 号 & $\underset{N}{N}$ \\
\hline & \multirow{3}{*}{$\stackrel{\llcorner}{\sim}$} & 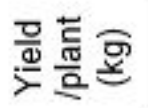 & $\bar{\sigma}$ & $\stackrel{m}{\circ}$ & $\stackrel{ }{\dddot{2}}$ & + & ํㅜㅇ & $\stackrel{\varphi}{\circ}$ & $\bar{\infty}$ & ণे & के & कृ & ๗ี \\
\hline & & 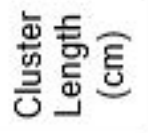 & $\begin{array}{l}\sigma \\
\infty\end{array}$ & ๗ँ & कृ & $\dddot{m}$ & $\underset{⿱ 亠}{\sigma}$ & $\underset{\infty}{\infty}$ & $\hat{\sigma}$ & $\begin{array}{l}\infty \\
\infty \\
\infty\end{array}$ & $\stackrel{\varphi}{\sigma}$ & $\stackrel{\varphi}{\dot{I}}$ & $\stackrel{m}{\sim}$ \\
\hline & & 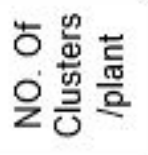 & 窝 & $\stackrel{ }{\sim}$ & ๗ె & 유 & $\stackrel{\text { N }}{ }$ & 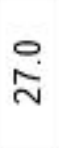 & $\stackrel{ }{N}$ & $\stackrel{0}{\sim}$ & $\stackrel{\circ}{N}$ & $\stackrel{ }{\check{1}}$ & $\stackrel{J}{\sim}$ \\
\hline & ¿ั. & हे & $N$ & $\stackrel{n}{m}$ & $\stackrel{0}{+}$ & $N$ & $\stackrel{m}{m}$ & $\stackrel{0}{\forall}$ & $N$ & $\stackrel{n}{m}$ & $\stackrel{8}{\forall}$ & & \\
\hline \multicolumn{3}{|c|}{ 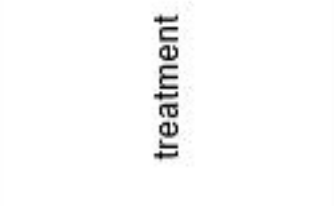 } & \multicolumn{3}{|c|}{$\begin{array}{l}\overline{\overline{0}} \\
\frac{0}{0} \\
\text { 心 }\end{array}$} & \multicolumn{3}{|c|}{ 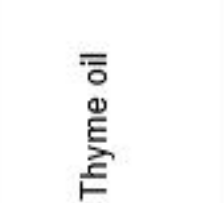 } & \multicolumn{3}{|c|}{$\begin{array}{l}\overline{\overline{0}} \\
\text { 峷 }\end{array}$} & 흘 & . \\
\hline
\end{tabular}


and Godard (2009) mentioned that plant extracts inhibited the development of Plasmopara viticola at a significant degree. Pereira et al., (2010) used plant extracts beside traditional fungicides for the protection of vine against downy mildew. Harm et al., (2011) verified the induced resistance to control downy mildew of grape vine plants which was determined by the increase of pathogenesis_related proteins Rawal (2013) and torre et al., (2014) revealed that plant extracts and essential oils were able to control the development of grapes downy mildew. Anelkovic et al., (2015) noticed that total phenolic content was higher in healthy grapevine leaf extracts than infected ones. The first was richer in flavonls, phenolic acid and flavan -3 - ols. Naik et al., (2015) noticed enhansments of chlorophylls, carotenoids and total polyphenols in grape leaves infected with downy mildew after application of Agava cantala leaf extract. They also noticed that peroxidase and polyphenol oxidase activities were increased and the activity of catalase enzyme was decreased in sprayed grapevine leaves Such observations clear that plant extracts and /or plant oils may be useful for controlling downy mildew disease of grapes. Mode of action of these compounds may be due to inhibition of the indirect sporangia germination, inhibition of the fungus development, enhancement of chlorophylls, carotenoids with total polyphenols, secretion of fungicidal compounds, increase of pathogenesisrelated proteins and phenolic compounds.

\section{REFERENCES}

Achimu, P. and E. Schlosser (1992). Effect of neem seed extracts (Azadirachta indica A. Juss) against downy mildew (Plasmopara viticola) of grapevine. Mededelingen van de Faculteit Land bouw wetens chappen, Universiteit Gent; 57(2b):423-431.

Anelkovic, M., B. Radovanovic, A.M. Anelkovic and V. Radovanovic (2015). Phenolic compounds and bioactivity of healthy and infected grapevine leaf extracts from red Varieties Merlot and Vranac (Vitis vinifera L.). Plant Foods for Human Nutrition; 70(3):317-323.

Berkelmann-Lohnertz, B. (2002). Copper replacement in organic viticulture - state of the art in legislation and research. 10th International Conference on Cultivation Technique and Phytopathological Problems in Organic Fruit-Growing and Viticulture. Proceedings of a conference, Weinsberg, Germany, 4-7 February 2002:123-126.

Cohen, Y., A. Baider, B. Ben-Daniel and Y. Ben-Daniel (2002). Fungicidal preparations from Inula viscosa. Plant Protection Science;:629-630.

Chovelon, M. (2006). Efficacy of salicylic acid against Plasmopara viticola. Bulletin OILB/SROP; 29(11):27-32.

Cohen, Y., Wang WenQiao, B. H. BenDaniel and Y. Ben-Daniel (2006). Extracts of Inula viscosa control downy mildew of grapes caused by Plasmopara viticola. Phytopathology; 96(4):417-424.

Chen Jiao, Dai GuangHui, Gu ZhenFang and Miao YiFang (2002). Inhibition effect of 58 plant extracts against grape downy mildew (Plasmopara viticola). [Chinese] Natural Product Research and Development; 14(5):9-13.

Dai, G. H., C. Andary, L. Cosson-Mondolot and D. Boubals (1994). Polyphenols and resistance of grapevines to downy mildew. Acta Horticulturae; (381(II)):763766.

Dagostin, S., T. Formolo and I. Pertot (2008). Replacement of copper in organic Viticulture: efficacy evaluation of new natural fungicides against downy mildew IOBC/WPRS Bulletin;. 36:87-90.

Dagostin, S., T. Formolo, O. Giovannini, A. Schmitt and I. Pertot (2009). Antifungal activity of Salvia officinalis extract against Plasmopara viticola. OBC/WPRS Bulletin; 43:217-220. 
Dagostin, S., T. Formolo, O. Giovannini, I. Pertot and A. Schmitt (2010). Salvia officinalis extract can protect grapevine against Plasmopara viticola. Plant Disease;. 94(5):575-580.

Fadeel, A.A. (1962). Location and properties of chloroplasts and Pigments determination in shoots. Plant Physiol., 119:975-986.

Gosev, N.A. (1966). Some methods in studying plant water relation leningrade. Acad. of Science.

Gindro, K. and S. Godard (2009). Is it possible to induce grapevine defence mechanisms?: A new method to evaluate the potential of elicitors. [French] Progres Agricole et Viticole;. 126(23):553-558.

Harm, A., H. H. Kassemeyer, T. Seibicke and F. Regner (2011). Evaluation of chemical and natural resistance inducers against downy mildew (Plasmopara viticola) in grapevine. American Journal of Enology and Viticulture; 62(2):184-192

Kast, W. K.; Bleyer, K.(2006):Effects of plant extracts on downy mildew of vine.Bulletin OILB/SROP; 29(11): 37-40.

Kalapos, T. (1994). Leaf water potential , leaf water deficit relationship for ten species of semiarid grassland community. Plant and soil, 160:105-112.

Klinkenberg, H. J., R. Stierl and H. W. Dehne (1998). Investigations on fungicide resistance in oomycetes. Mededelingen

FaculteitLandbouwkundige en Toegepaste Biologische Wetenschappen, Universiteit Gent; 10091015.

Leite, C. D., R. V. Botelho, C. M. D. R. Faria and A. J. Maia (2011). Garlic extract and vegetable oil in the control of downy mildew in grapevines. [Portuguese] Revista Brasileira de Fruticultura; 429436.

Molitor, D., D. Heibertshausen, O. Baus, B. Loskill, M. Maixner and B. Berkelmann-
Lohnertz (2010). Use of a Sapindus mukorossi-extract to regulate fungal pathogens on vines - an alternative strategy for organic grape protection? [German] Journal fur Kulturpflanzen; 444450.

Naik, V. V., M. S. Bansode and S. P. Bartakke (2015). Foliar application of Agave cantala Roxb. leaf extract enhances antioxidative defense mechanism in grape (Vitis vinifera L.) leaves infected with downy mildew.

World Journal of Pharmaceutical Research; 2039-2056.

Pereira, V. F., M. L. V. de Resende, A. C. A. Monteiro, P. M. Ribeiro Junior, M. de A. Regina and F. C. L. Medeiros (2010): Alternative products for the protection of vine against downy mildew. [Portuguese] Pesquisa Agropecuaria Brasileira; 2010. 45(1):25-31.

Robotic, V., R. Bosancic and M. Mojic (2000). Controlling vine powdery and downy mildews with the urticum preparation. IFOAM 2000 - the world grows organic. Proceedings, 6th International Congress on Organic Viticulture, Convention Center, Basel, Switzerland, 25-26 August 2000; 193194.

Rawal, R. D. (2013). Present scenario of important fungal diseases of grapes and their management. Indian Phytopathology; 1-9

Rajeswari, E., K. Chitra, K. Seetharaman and V. Sankaralingam (2008). Exploiting medicinal plants and phylloplane microflora for the management of grapevine downy mildew.

Archives of Phytopathology and Plant Protection; 213-220.

Schilder, A. M. C., J. M. Gillett, R. W. Sysak and J. C. Wise (2002). Evaluation of environmentally friendly products for control of fungal diseases of grapes. 10th International Conference on Cultivation 
Technique and Phytopathological Problems in Organic Fruit-Growing and Viticulture Proceedings of a conference, Weinsberg, Germany: 163-167.

Schmitt, A., S. Kunz, S. Nandi, B. Seddon, A. Ernst (2002). Use of Reynoutria sachalinensis plant extracts, clay preparations and Brevibacillus brevis against fungal diseases of grape berries. 10th International Conference on Cultivation Technique and Phytopathological Problems in Organic Fruit-Growing and Viticulture. Proceedings of a conference, Weinsberg, Germany, 4-7 February 2002; 146-151.
Scannavini, M., A. Barani, A. Franchi and P. Bortolotti (2004). Use of a plant growth regulator for control of downy mildew on grape. [Italian] Informatore Agrario; 60(27): 68-70.

Soliman, N. K., M. S. Mikhaili; P. K. Harb and E. M. Khalil (1988). Response of broad bean plants infected with Rhizoctonia solani to application of growth regulators and calcium. Egypt. J. Phytopathol., 20 (1): 1 - 11.

Torre, A. la, C. Mandala, L. Pezza, F. Caradonia and V. Battaglia (2014). Evaluation of essential plant oils for the control of Plasmopara viticola. Journal of Essential Oil Research; 26(4):282-291. 


\section{M. Ammar, et al.,}

تأثير بعض المستخلصات والزيوت النباتية على مرض البياض الزغبى فى العنب

محمد محمد بيومى عمار ، جمعه عبد العليم عامر، أحمد محمد عبدالعال ،

أحمد اسماعيل بدوى جاعيل

قسم النبات الزراعى - كلية الزراعة - جامعة المنوفية - مصر

الملخص العربى

أجريت هذه الدراسة عامى 10 • بو 1 ـ ـ للتوصل إلى طرق صديقة للبيئة لمكافحة مرض البياض الزغبى فى

العنب • وأظهرت النتائج أن المستخلصات النباتية المائية لنباتات الخروع والدفله والنعناع تؤدى إلى نقص معنوى لنسبة وشدة الإصابة بالمرض وتزيد محتوى الأوراق من كلوروفيل أ؛ ب والكاروتين وتحسن العلاقات المائية للنبات. كما أدت المعاملات إلى الزيادة المعنوية فى عدد عناقيد العنب المنتجة للنبات وكذلك متوسط طول العنقود ومتوسط إنتاج النبات من الثمار مقارنة بالنبتات الغير معاملة ـ وكان الخروع هو أفضل هذه المستخلصات ـ ـ كما أدت المعاملة بمستخلصات الزيوت العطرية للخروع والزعتر والنعناع إلى نفس النتائج المشار إليها سابقا.

أسماء السادة المحكمين

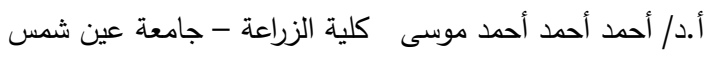

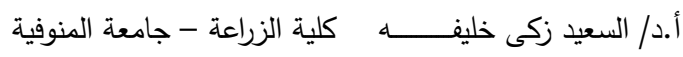

